\title{
CLOSABLE OPERATORS AND SEMIGROUPS
}

\author{
NEIL FALKNER
}

\begin{abstract}
We show that a linear operator is closable iff it is possible to weaken the topology on its range in a certain nice way so as to render the operator continuous. We apply this result to show that if $E$ is a sequentially complete locally convex Hausdorff space and $(L(t))_{0<1<\infty}$ is a locally equicontinuous semigroup of class $\left(C_{0}\right)$ in $E$ with generator $S$ and if $x \in E$ (not necessarily belonging to the domain of $S$ ) then the function $u(t)=L(t) x$ is a solution. in a generalized sense, of the initial value problem $u^{\prime}(t)=S u(t), u(0)=x$, and that such a generalized solution is unique. It should be noted here that $u(t)$ may fail to belong to the domain of $S$ so we must assign a suitable meaning to the expression $S u(t)$.
\end{abstract}

THEOREM 1. Let $E$ and $F$ be topological vector spaces and let $S$ be a linear operator from $E$ into $F$ with domain $D \subseteq E$. Then $S$ is closable iff there is a Hausdorff linear topology $\tau$ on $F$ which is weaker than the original topology on $F$ and which renders $S$ continuous. In this case, there is a canonical description of the strongest such topology $\tau$.

Proof. As is usual, we identify $S$ with its graph. Define $\psi: D \times F \rightarrow F$ by $\psi(x, y)=y-S x$. Then $\psi$ is a linear map, the kernel of $\psi$ is $S$, and the range of $\psi$ is all of $F$. Let $\tau$ be the quotient topology induced on $F$ by $\psi$. Then it is easy to check that $\tau$ is the strongest linear topology on $F$ which is weaker than the original topology of $F$ and which renders $S$ continuous. Thus we need only show that $S$ is closable (as an operator from $E$ to $F$ ) iff $\tau$ is Hausdorff. But, as is well known, $S$ is closable iff $S$ is closed in $D \times F$, and $\tau$ is Hausdorff iff the kernel of $\psi$, namely $S$, is closed in $D \times F$. This completes the proof.

Theorem 2. Let $E, F, D$, and $S$ be as in Theorem 1. Assume $D$ is dense in $E$. Suppose $S$ is closable and let $\tau$ be the strongest linear topology on $F$ which is weaker than the original topology of $F$ and which renders $S$ continuous. Let $G$ be the completion of $(F, \tau)$, let $T$ be the unique extension of $S$ to a continuous linear map of $E$ into $G$, and let $\bar{S}$ be the closure of $S$ in $E \times F$. Then $T \supseteq \bar{S}$, and if $x \in E$ and $T x \in F$ then $x$ is an element of the domain of $\bar{S}$.

Proof. It is clear that $T \supseteq \bar{S}$. Suppose $x \in E$ and $y=T x \in F$. Let $\left(x_{i}\right)$ be a net in $D$ which converges in $E$ to $x$. Let $U$ and $V$ be neighbourhoods of 0 in $E$ and $F$ respectively. Let $W=\psi[(U \cap D) \times V]$ where $\psi$ is as in the proof of Theorem 1 . As $\psi$ is linear and $\tau$ is the quotient topology induced on $F$ by $\psi, \psi$ is open onto $(F, \tau)$ so $W$ is a neighbourhood of 0 in $(F, \tau)$. Now $\left(y-S x_{i}\right) \tau$-converges to 0 . Thus there exists $i_{0}$ such that $i \geqslant i_{0}$ implies $\psi\left(x_{i}, y\right) \in W$; i.e., $\left(x_{i}, y\right) \in[(U \cap D) \times V]+S$.

Received by the editors March 18, 1982.

1980 Mathematics Subject Classification. Primary 47A05, 47D05.

(c) 1983 American Mathematical Society 0002-9939/82/0000-0427/\$01.75 
Hence $(x, y) \in \overline{(U \times V)+S}$. But $\bar{S}$ is the intersection of the sets of the form $(U \times V)+S$ where $U$ and $V$ range over neighbourhoods of 0 in $E$ and $F$ respectively. Thus $(x, y) \in \bar{S}$. Hence $x$ is an element of the domain of $\bar{S}$. This completes the proof.

Now let $E$ be a sequentially complete locally convex Hausdorff topological vector space, which will be fixed throughout the rest of the discussion. We shall give some applications of Theorems 1 and 2 to the study of semigroups of operators on $E$. Let $(L(t))_{0<i<\infty}$ be a locally equicontinuous semigroup of class $\left(C_{0}\right)$ on $E$. We recall that this means:

(a) Each $L(t)$ is a continuous linear operator on $E$.

(b) $L(0)=I$ and $L(s+t)=L(s) L(t)$ for all $s$ and $t$.

(c) For each $x \in E$, the map $t \mapsto L(t) x$ is continuous on $[0, \infty)$.

(d) For any $a \in[0, \infty),\{L(t): 0 \leqslant t \leqslant a\}$ is equicontinuous.

Let $S$ be the generator of $(L(t))$. Then $S$ is a closed, densely defined, linear operator in $E$. (The proof given in [1] for this standard result in the case where $E$ is a Banach space can easily be generalized to the present setting.) Let $D$ be the domain of $S$ and let $\tau$ be the strongest linear topology on $E$ which is weaker than the original topology on $E$ and which renders $S$ continuous from $D$ with its original topology to $(E, \tau)$. By Theorem 1, $\tau$ is Hausdorff because $S$ is closed. Also, by the description of $\tau$ given in the proof of Theorem $1, \tau$ is locally convex. Let $F$ be the completion of $(E, \tau)$ (then $F$ is also locally convex) and let $T$ be the unique extension of $S$ to a continuous linear map of $E$ into $F$.

It is natural to ask whether $(L(t))$ can be extended to a semigroup on $F$ and what properties this extended semigroup may have. The next result deals with this question.

THEOREM 3. The following statements hold:

(a) For each $t \in[0, \infty)$, there is a unique continuous linear operator $M(t)$ on $F$ whose restriction to $E$ is $L(t)$.

(b) $(M(t))$ is a locally equicontinuous semigroup of class $\left(C_{0}\right)$ on $F$ and the generator $A$ of $(M(t))$ is an extension of $T$.

(c) If $E$ is complete and if for some real number $\beta,\left\{e^{-t \beta} L(t): 0 \leqslant t<\infty\right\}$ is equicontinuous on $E$, then $A=T$.

(We remark that if $E$ is a Banach space then the hypotheses of (c) are automatically satisfied; see [3, p. 232].)

Proof. Let $\psi$ be the map $(x, y) \mapsto y-S x$ from $D \times E$ onto $E$. It is a well-known observation that if $x \in D$ then for $0 \leqslant t<\infty$ we have $L(t) x \in D$ and $S L(t) x=$ $L(t) S x$. From this it follows that $L(t) \psi(x, y)=\psi(L(t) x, L(t) y)$. Suppose $a \in$ $(0, \infty)$ and $W$ is any neighbourhood of 0 in $(E, \tau)$. Then there are neighbourhoods $U$ and $V$ of 0 in $E$ such that $\psi[(D \cap V) \times V] \subseteq W$ and $L(t)[U] \subseteq V$ for $0 \leqslant t \leqslant a$. But then $L(t)[\psi[(D \cap U) \times U]] \subseteq W$ for $0 \leqslant t \leqslant a$. Now $\psi[(D \cap U) \times U]$ is a neighbourhood of 0 in $(E, \tau)$ since $\psi$ is open onto $(E, \tau)$. Thus $\{L(t): 0 \leqslant t \leqslant a\}$ is an equicontinuous family of linear operators on $(E, \tau)$. Part (a) follows from this. Moreover, since the closure in $F$ of any neighbourhood of 0 in $(E, \tau)$ is a 
neighbourhood of 0 in $F$, it follows that $\{M(t): 0 \leqslant t \leqslant a\}$ is equicontinuous on $F$ for any $a \in(0, \infty)$. (A similar argument shows that under the hypotheses of (c), $\left\{e^{-t \beta} M(t): 0 \leqslant t<\infty\right\}$ will be equicontinuous on $F$.) That $M(0)=I$ and $M(s+t)$ $=M(s) M(t)$ is clear. The continuity of $t \mapsto M(t) x$ for $x \in F$ is proved by a uniform convergence argument using the local equicontinuity of $(M(t))$. It is obvious that $A \supseteq S$. Suppose $x \in E$ and $y=T x$. Then there is a net $\left(x_{i}\right)$ in $D$ converging to $x$ in $E$. Then $S x_{i} \rightarrow y$ in $F$ and $x_{i} \rightarrow x$ in $F$. As $A \supseteq S$ and $A$ is closed in $F \times F, x$ belongs to the domain of $A$ and $A x=y$. Thus $A \supseteq T$.

Now suppose the hypotheses of part (c) are satisfied. Let $\lambda \in(\beta, \infty)$. Then $(\lambda-S)^{-1}$ exists and is continuous from $E$ into $E$ and $R:=(\lambda-A)^{-1}$ exists and is continuous from $F$ into $F$; see [3, p. 240]. Since $A \supseteq S, R \supseteq(\lambda-S)^{-1}$. It is easy to check that $(\lambda-S)^{-1} \circ \psi$ is continuous from $D \times E$ into $E$. It follows that $(\lambda-S)^{-1}$ is continuous from $(E, \tau)$ into $E$. Therefore the range of $R$ is contained in $E$, since $E$ is complete. Hence $A=T$. This completes the proof.

If $x \in D$ then $u(t)=L(t) x$ is the unique solution of the initial value problem $u^{\prime}(t)=S u(t), u(0)=x$. As the next result shows, this is actually true for any $x \in E$ and not just for $x \in D$, provided we replace $S$ by its extension $T$ and compute the derivative $u^{\prime}(t)$ relative to the topology of $F$ instead of $E$.

THEOREM 4. The following statements hold:

(a) For each $x \in E$, we have $F-d L(t) x / d t=T L(t) x$ on $[0, \infty)$.

(b) If $a \in(0, \infty), x \in E$, and $u:[0, a] \rightarrow E$ is continuous from $[0, a]$ to $F$ and satisfies $F-d u(t) / d t=T u(t)$ on $(0, a)$ and $u(0)=x$ then $u(t)=L(t) x$ for $0 \leqslant t \leqslant$ a.

Proof. Let $(M(t))$ and $A$ be as in Theorem 3. Then $L(t) x=M(t) x$ and $T L(t) x=A L(t) x$. Thus (a) just says $F-d M(t) x / d t=A M(t) x$ which, as is well known, holds for all $x$ in the domain of $A$ and in particular for all $x \in E$.

To prove (b), consider any $b \in(0, a]$. Define $y:[0, b] \rightarrow E$ by $y(t)=$ $L(b-t) u(t)=M(b-t) u(t)$. Using the equicontinuity of $\{M(s): 0 \leqslant s \leqslant b\}$ on $F$, one can easily check that $y$ is continuous from $[0, b]$ into $F$ and $F-d y(t) / d t=0$ on $(0, b)$. Hence $y(b)=y(0)$; i.e., $u(b)=L(b) u(0)=L(b) x$. This completes the proof.

As a final application we give an alternative proof of a result of $\mathrm{K}$. Yosida [2, $\mathrm{p}$. 58 , to the effect that the weak generator of $(L(t))$ is the same as $S$.

THEOREM 5. Suppose $x, y \in E$ and

$$
w-\lim _{h \downarrow 0} \frac{L(h)-I}{h} x=y,
$$

where $w$-lim denotes weak limit in $E$. Then $x \in D$ and $y=S x$.

Proof. The hypotheses implies that

$$
F-w-\lim _{h \downarrow 0} \frac{M(h)-I}{h} x=y
$$


where $M$ is as in Theorem 3 and $F$-w-lim denotes weak limit in $F$. But by (b) of Theorem 3,

$$
F-\lim _{h \downarrow 0} \frac{M(h)-I}{h} x=T x
$$

Since $F$ is locally convex and Hausdorff, its weak topology is also Hausdorff. Hence $y=T x$. Since $S$ is closed and $T x \in E$, we have $x \in D$ by Theorem 2 . Hence $y=S x$. This completes the proof.

\section{REFERENCES}

1. W. Rudin, Functional analysis, McGraw-Hill, New York, 1973.

2. K. Yosida, Lectures on semi-group theory and its application to Cauchy's problem in partial differential equations, Tata Institute of Fundamental Research, Bombay, 1957.

3. __ Functional analysis, 5th ed., Springer-Verlag, Berlin. Heidelberg and New York, 1978.

Department of Mathematics, The Ohio State University, Columbus, Ohio 43210

Current address: Department of Mathematics, Stanford University, Stanford, California 94305 